Wim Naudé*

\title{
Intelligente Eindämmungsstrategien gegen Covid-19: Die Rolle von Künstlicher Intelligenz und Big Data
}

https://doi.org/10.1515/pwp-2020-0021

Zusammenfassung: Die Covid-19-Pandemie ist eine Gesundheits- und eine Wirtschaftskrise. Die politischen Reaktionen auf beide sind aufgrund der durch fehlende $\mathrm{Da}$ ten verursachten Unsicherheit nicht optimal. Der Mangel an Daten schränkt den Einsatz von Künstlicher Intelligenz (KI) und die Genauigkeit epidemiologischer Modelle ein. Infolgedessen ist die Künstliche Intelligenz noch nicht in der Lage, bei der Vorhersage, dem Tracking und der Diagnose von Covid-19-Infektionen wirklich zu helfen. Der Mangel an Trainingsdaten für die KI schränkt die Verwendung von datenschutzsensitiven Tracing-Apps weiter ein. Wim Naudé kommt zu dem Schluss, dass das Sammeln ausreichender und geeigneter, unverzerrter Daten, gewonnen auch aus Apps und groß angelegten diagnostischen Tests, eine Voraussetzung für die Verbesserung der Strategien zur Bewältigung der Zwillingskrisen ist. Angesichts der exorbitanten wirtschaftlichen Kosten der bisher angewandten ziemlich groben Eindämmungsmaßnahmen werden „intelligente“ Eindämmungsstrategien, die auf einer besseren Datenanalyse beruhen, die Wiederaufnahme der wirtschaftlichen Tätigkeit ermöglichen und verhindern, dass es zu weiteren Infektionswellen kommt. Den Datenschutz zu garantieren und öffentliches Vertrauen zu den Datenanalyse- und KI-Systemen intelligenter Eindämmungsstrategien aufzubauen, ist jetzt noch dringlicher als je zuvor.

JEL-Klassifikation: O32, O39, I19, 020

Schlüsselwörter: Künstliche Intelligenz, Covid-19, Corona-Virus, Gesundheit, Datenwissenschaft, Wirtschaftspolitik, Innovation

*Kontaktperson: Wim Naudé, Maastricht School of Management, Maastricht, Niederlande, und RWTH Aachen, Technologie- und Innovationsmanagement (TIM), Fakultät für Wirtschaftswissenschaften, Kackertstraße 7, 52072 Aachen,

E-Mail: naude@time.rwth-aachen.de

\section{Einführung}

Die Antwort auf die Covid-19-Pandemie ist letztlich eine wissenschaftlich-technische, insbesondere die Suche nach einem Impfstoff und nach Therapien. Bis dahin muss die Politik die Ausbreitung der Krankheit eindämmen und verlangsamen. In der Tat muss die epidemiologische Kurve so weit wie möglich „abgeflacht“ werden (Anderson et al. 2020). Eine solche Politik umfasst auch nicht-pharmazeutische Interventionen (NPI), zum Beispiel Lockdowns und soziale Distanzierung, die dramatische und sogar katastrophale wirtschaftliche Auswirkungen haben werden, indem sie wirtschaftliche und gesellschaftliche Aktivitäten einschränken. Gemäß dem World Economic Outlook des IWF wird das globale Bruttoinlandsprodukt im Jahr 2020 um 3 Prozent schrumpfen, wobei der kumulierte Verlust an globalem BIP in den Jahren 2020 und 2021 rund 9 Billionen Dollar betragen könnte (Gopinath 2020). Schätzungen von Eichenbaum et al. (2020) für die Vereinigten Staaten deuten darauf hin, dass eine optimale Lockdown-Politik die Zahl der Todesfälle um 500.000 verringert, aber zu einem Rückgang des durchschnittlichen Verbrauchs um 22 Prozent im ersten Jahr führen wird.

Je länger die Regierungen diese Art von Politik durchsetzen, desto größer wird der wirtschaftliche Schaden sein. Daher ringen alle Regierungen mit Strategien für einen „Ausstieg“ aus diesen Maßnahmen. Da es keinen Impfstoff und keine einschlägigen Erfahrungen mit einer solchen Pandemie gibt, ist die Politik erheblichen Unsicherheiten und Risiken ausgesetzt. Ist die „Kur“ schlimmer als die Krankheit? Was ist der optimale Trade-off zwischen gesundheitlichen und wirtschaftlichen Schäden?

Was die Beantwortung dieser Fragen noch schwieriger macht, ist die Tatsache, dass es einen robusten langfristigen Zusammenhang zwischen Armut, schlechter Gesundheit und Sterblichkeit gibt. Der mögliche dauerhafte Verlust der Lebensgrundlagen als Folge der Eindämmungspolitik kann im Laufe der Zeit zu einer höheren Sterblichkeit führen ${ }^{1}$.

1 Es lässt sich noch nicht abschließend feststellen, dass die derzeitigen Eindämmungsmaßnahmen aufgrund der höheren Arbeitslosig- 
Angesichts des Risikos, vom Regen in die Traufe zu kommen, bleibt den politischen Entscheidungsträgern kaum etwas anderes übrig, als auf die Technologie und insbesondere auf die Datenwissenschaft zurückzugreifen. Denn der Schlüssel zur Lösung dieses politischen Dilemmas liegt in der Überwindung des Datenmangels. So ist beispielsweise nicht bekannt, wie viele Menschen infiziert sind oder waren (Li et al. 2020); es ist nicht bekannt, wie ansteckend und wie tödlich das Virus ist (zum Beispiel Jelnov 2020). Es ist auch nicht bekannt, wie angemessen die in epidemiologischen Modellen verwendeten Annahmen über kritische Parameter sind (Anderson et al. 2020).

Ökonomen sind sich offenbar darin einig, dass eine Art von Ausstiegsstrategie auf umfangreichen diagnostischen Tests (also auf Datenerhebung) basieren wird, damit diejenigen Menschen, die entweder infektionsfrei oder immun sind, ihre wirtschaftliche Tätigkeit so schnell wie möglich wieder aufnehmen können (Dewatripont et al. 2020 und Eichenbaum et al. 2020). Ein anderer Ansatz für eine Exit-Strategie könnte darin bestehen, genauer zu bestimmen, wie ansteckend und tödlich das Virus ist: Wenn sich herausstellt, dass es sehr ansteckend ist, aber eine geringe Todesrate aufweist ${ }^{2}$, würde der Exit-Ansatz darin bestehen, in Zukunft nur diejenigen zu isolieren, die einem sehr hohen Risiko ausgesetzt sind, zum Beispiel alte Menschen. Wenn man mehr wüsste, wäre es einfacher, intelligente Eindämmungsstrategien ins Werk zu setzen, im Gegensatz zu den groben Maßnahmen, mit denen in den meisten Ländern derzeit im Wesentlichen fast alle Bürger unter Quarantäne gestellt werden.

Intelligente Eindämmungsmaßnahmen werden aus einer Kombination von künstlicher Intelligenz (KI), Datenerfassung und Diagnosetests sowie Mobilfunktechnologie bestehen. Diese Kombination wird die Suche nach einem Impfstoff erleichtern, kann dazu beitragen, die Infektion schneller zu diagnostizieren, Eindämmungsmaßnahmen

keit oder der Unzugänglichkeit überlasteter Gesundheitseinrichtun gen zu einem langfristigen Anstieg der Sterblichkeit führen werden. Kurzfristig gesehen können die Maßnahmen vielmehr sogar die Sterblichkeit verringern, aufgrund anderer, nicht krankheitsbedingter Ursachen wie Verkehrsunfällen und Kriminalität. Es hat sich beispielsweise herausgestellt, dass kurzfristig eine antizyklische Beziehung zwischen der Sterblichkeit und dem Konjunkturzyklus besteht (Ballester et al. 2019).

2 Beispielsweise liefert Razavian (2020) Schätzungen, die darauf hindeuten, dass Covid-19 300-mal so ansteckend wie die saisonale Grippe, aber viel weniger tödlich ist (mit einer Fallsterblichkeitsrate Case Fatality Rate (CFR) - von 0,04 Prozent) und dass etwa 96 Prozent der Menschen infiziert werden, aber keine Symptome zeigen. Li et al. (2020) beziffern den Anteil der asymptomatischen Bevölkerung auf 86 Prozent. durchzusetzen und diese in einer Ausstiegsstrategie eben auch zu lockern.

Das Problem mit der KI indes ist, dass es sich um eine relativ teure Technologie handelt. Sie ist nicht allgemein zugänglich, und ihre Entwicklung und ihr Besitz sind sehr konzentriert (Naudé 2019). Ein Teil des Problems sind die Daten: Die Erstellung zuverlässiger KI-Modelle erfordert große Mengen unverzerrter und genauer Daten. Datengetriebene Netzwerkökonomien erzwingen „Winnertakes-all“-Effekte und Marktkonzentrationseffekte, was wiederum das Schreckgespenst aufkommen lässt, dass die Vorteile der KI eher wenigen als vielen Menschen zugutekommen werden. Bei der Bekämpfung einer globalen Pandemie, in der die Kosten unverhältnismäßig stark von den Armen und Marginalisierten getragen werden, ist dies nicht der wünschenswerte Ansatz.

Darüber hinaus können die Durchsetzung von Lockdown-Beschränkungen und das Management ihrer Lockerung durch Tracing-Apps erfordern, dass die Bürger datenschutzbezogene Grundrechte aufgeben, was die Aussicht auf eine schleichende Ausweitung des Staatseinflusses erhöht, sobald die Regierungen Zugang zu großen Möglichkeiten der digitalen Überwachung haben. Wenn dies geschieht, besteht die Gefahr, dass die Menschen das Vertrauen zu den Regierungen verlieren und dass dann die Gesundheitspolitik schwieriger wird (Ienca und Vayena 2020).

Die KI kann insofern im Kampf gegen Covid-19 eine wertvolle Rolle spielen, als sie hilft, die wirtschaftlichen und gesundheitlichen Kosten zu senken. Wenn sie jedoch nicht richtig gehandhabt wird, wenn politische Fehler unterlaufen, dann kann sie der Volkswirtschaft auch hohe, langfristige Kosten in Form von höherer Ungleichheit, langsamer wirtschaftlicher Erholung und geringerer Dynamik aufbürden. Sie kann die wahrscheinlichen schädlichen strukturellen Folgen der Pandemie verstärken, beispielsweise die beschleunigte Automatisierung der Produktion, die Rückverlagerung der wirtschaftlichen Aktivitäten aus dem Ausland und die Konsolidierung der Marktmacht großer digitaler Plattformunternehmen.

Wie die KI verwendet wird und wie sie im Kampf gegen Covid-19 eingesetzt werden sollte, sind daher Fragen von größter Bedeutung für Ökonomen. Der Rest dieses Beitrags bietet aus wirtschaftspolitischer Sicht einen kurzen Überblick über die potenzielle Rolle der KI und der Technologien zur Verarbeitung und Auswertung riesiger Datenmengen (Big Data) für intelligente Eindämmungsstrategien im Kampf gegen Covid-19.

Der Aufsatz ist wie folgt aufgebaut. In Abschnitt 2 gebe ich eine kurze Einführung in die KI, unter Betonung der Abhängigkeit von Daten. Dann erörtere ich in Abschnitt 3 
die Covid-19-Pandemie, wobei der Schwerpunkt auf der Suche nach einem Impfstoff und auf dem Einsatz von NPI liegt, beides Bereiche, in denen Daten im Mittelpunkt stehen. Abschnitt 4 ist dem Einsatz der KI in pharmazeutischen Interventionen bei Diagnosen, Prognosen und der Suche nach einem Impfstoff und nach Therapien gewidmet. In Abschnitt 5 befasse ich mich mit dem Einsatz der KI bei NPI, insbesondere bei der Vorhersage von Infektionen und der sozialen Kontrolle. Abschnitt 6 schließt mit einer Zusammenfassung und mit Empfehlungen sowohl für eine intelligente Eindämmungspolitik als auch für weitere Forschung.

\section{Künstliche Intelligenz}

Intelligenz ist ein schwierig zu definierendes Konzept. Wie Tegmark (2017, S. 49) hervorhob, gibt es selbst unter intelligenten Intelligenzforschern keine Einigkeit darüber, was Intelligenz ist. Daher überrascht es nicht, dass es auch keine allgemein akzeptierte Definition der Künstlichen Intelligenz gibt (Van de Gevel und Noussair 2013). Für die Zwecke hier wird sich der Begriff KI auf die Verwendung von maschinellem Lernen (ML), der Verarbeitung natürlicher Sprache (Natural Language Processing - NLP) und Computer Vision (CV) beziehen, um Computer dazu zu bringen, Vorhersagen zu treffen und Muster zu erkennen. Alle KI-Anwendungen sind entweder Vorhersagemaschinen oder Mustererkenner. Bemerkenswerte Beispiele sind selbstfahrende Fahrzeuge (die Computer Vision und ML zur Erkennung von Objekten und zur Vorhersage des Fahrerverhaltens verwenden), virtuelle Assistenten wie Siri oder Alexa (die auf NLP angewiesen sind) und Empfehlungssysteme, wie sie von Netflix oder Amazon verwendet werden, um Verbraucherpräferenzen und Kaufverhalten vorherzusagen (unter Verwendung von ML).

Wenn sie in diesen Anwendungen Vorhersagen machen und Muster erkennen, sollen Computer ,intelligent handeln“ (New Scientist 2017, S. 3). Natürlich sind sie nicht intelligent, und in dieser Hinsicht ist KI eine Fehlbezeichnung. Darüber hinaus sind alle Handlungen, die intelligent erscheinen, sehr bereichsspezifisch. Der Aufbau eines KI-Modells erfordert Trainingsalgorithmen auf bestimmten Datensätzen. KI-Modelle wie „AlphaGo“"3, die Spiele spielen, werden auf Spieldaten trainiert und können nur Spiele spielen. Ebenso kann ein KI-Modell, das vorhersagt, welchen Film man sich als nächstes ansehen möchte,

3 Für eine Erörterung des AlphaGo zugrundeliegenden General-Reinforcement-Learning-Algorithmus siehe Silver et al. 2018. nur das und nichts anderes. Es gibt keine Übertragung des Gelernten von einem Bereich auf einen anderen und auch keinen gesunden Menschenverstand. Deshalb wird KI manchmal als „enge“ KI bezeichnet, um sie von der Science-Fiction-Idee einer Künstlichen Allgemeinen Intelligenz (Artificial General Intelligence, AGI) zu unterscheiden, die das Lernen von einem Bereich auf einen anderen übertragen kann, oder von einer Superintelligenz, die über die menschliche Intelligenz hinausgeht.

Das wesentliche Merkmal ist hier die Abhängigkeit von Daten. Als der Begriff „Künstliche Intelligenz“ 1956 am Dartmouth College in den Vereinigten Staaten geprägt wurde, versuchten Wissenschaftler, KI mit Hilfe symbolischen Denkens aufzubauen (Moor 2006). Dieser Ansatz war weitgehend erfolglos. Erst nach der Schaffung des Internets, der Erzeugung großer Datenmengen durch Sensoren und mobile Geräte sowie der Möglichkeit des CloudComputing wurde eine KI möglich, die auf dem Lernen aus Daten basiert. $\mathrm{Zu}$ den Schlüsselbeiträgen $z u$ den Techniken von ML, die eine Nutzung großer Daten erleichterten, gehören Hinton und Salakhutdinov 2006 sowie Hinton et al. 2006.

Angesichts des Datenbedarfs der KI gibt es drei Implikationen, die für die gegenwärtigen Zwecke relevant sind. Erstens ist die Qualität der Daten von Bedeutung. Verzerrte und ungenaue Daten führen zu Fehlern bei Vorhersagen und Mustererkennung. Dies hat unter anderem zur sogenannten „Furchtbaren KI“ geführt, also zum Beispiel KIModelle, die rassistische und sexistische Ergebnisse erzeugen und die Profilerstellung, Diskriminierung und Verfolgung ethnischer Minderheiten erleichtern ${ }^{4}$. Zweitens würde die datengesteuerte Netzwerkökonomie zu „Winnertakes-all"-Effekten und einer Marktkonzentration durch einige wenige Großunternehmen führen, denen es gelingt, sich einen First-Mover-Vorteil beim Zugang zu Big Data zu verschaffen (siehe Farboodi et al. 2019). Infolgedessen dominieren nur relativ wenige große Akteure das Feld der KI-Entwicklung: 30 Firmen in drei Regionen (Nordamerika, EU und China) vereinigen mehr als 90 Prozent der Patentanmeldungen und der Beschaffung von Risikokapital in der KI auf sich (WIPO 2019). Drittens sind Daten wertvoller geworden. Infolgedessen ist das Datensammeln in den Vordergrund gerückt, was Auswirkungen auf den Datenschutz, die Cybersicherheit und die Überwachung hat (siehe beispielsweise Feldstein 2019 und Reardon et al. 2019).

4 Siehe Daos „Awful AI“-Liste auf GitHub, online verfügbar unter https://github.com/daviddao/awful-ai. 
Alle drei Auswirkungen - Verzerrungen, Marktkonzentration und Datenschutzverletzungen - sind zu zentralen gesellschaftlichen Anliegen geworden und heizen eine reichhaltige Debatte über die Governance der KI an. Für Ökonomen besteht in diesem Zusammenhang besonders das Interesse, einen Beitrag zum Verständnis der datengesteuerten Netzwerkökonomie zu leisten, unter anderem des digitalen und KI-Unternehmertums, der Preisgestaltung von Daten und der Fortentwicklung der Wettbewerbspolitik. Darüber hinaus müssen all diese Implikationen verstanden werden, wenn man darüber nachdenkt, wie KI am besten zur Bekämpfung von Covid-19 eingesetzt werden kann.

\section{Covid-19}

Covid-19 wird durch das SARS-CoV-2-Virus verursacht. Dieses wurde im Dezember 2019 in Wuhan, China, identifiziert. Am 11. März 2020 erklärte die Weltgesundheitsorganisation (WHO) Covid-19 zur globalen Pandemie. Das Virus wird als die ernsthafteste Bedrohung der öffentlichen Gesundheit beschrieben, die seit der „Spanischen Grippe“ von 1918 von einem Atemwegsvirus ausgeht (Ferguson et al. 2020, S. 1).

Eine Reihe nützlicher wissenschaftlicher Aufsätze ist veröffentlicht worden, in denen die Grundlagen der Krankheit zusammengefasst sind. Eine kurze Einführung mit regelmäßigen Aktualisierungen bietet das „Benutzerhandbuch zu Covid-19“" von Galeotti und Surico (2020). Eine umfangreiche Literatur zu allen Facetten der Krankheit breitet sich weiterhin rasch aus. Der „Covid-19 Evidence Navigator“5 von Grünwald et al. (2020) dokumentiert und kartographiert wissenschaftliche Publikationen, die Covid-19 gewidmet sind, und aktualisiert diese täglich aus PubMed. Daraus geht hervor, dass täglich Hunderte neuer Artikel veröffentlicht werden: Am 21. April 2020 wurden nicht weniger als 747 neue Publikationen verzeichnet. $\mathrm{Pu}$ blons $^{6}$ hat eine Online-Tabelle zur Verfügung gestellt mit „papers and pre-prints we've identified as being on the frontline of Covid-19 research“. Zum Zeitpunkt der Abfassung dieses Artikels (23. April 2020) waren insgesamt 8.097 Beiträge aufgelistet. Ein weiteres Beispiel ist Elsevier $^{7}$, der alle frühen und von Fachkollegen begutachteten Forschungsarbeiten zu Covid-19 sowie etwa 20.000 Covid-

5 Siehe https://gruenwald.shinyapps.io/covid19-evi/.

6 Siehe https://publons.com/publon/covid-19/?sort_by=date. 7 Siehe https://www.elsevier.com/connect/coronavirus-information-center. 19-bezogene Artikel auf ScienceDirect öffentlich zugänglich macht.

Angesichts der Tatsache, dass die Antwort auf die Covid-19-Pandemie letztlich eine wissenschaftlich-technische ist, kann man die Reaktion der wissenschaftlichen Gemeinschaft nur loben. Der Umfang der Arbeiten und die Schnelligkeit, mit der sie erscheinen, führt jedoch zu zwei Schwierigkeiten. Die eine besteht darin, dass die schiere Menge potenziell wichtiger oder nützlicher Forschungsergebnisse die Fähigkeit von Forschern und Forschungsteams stark strapaziert, diese $\mathrm{zu}$ analysieren und $\mathrm{zu}$ synthetisieren. Hier können Data-Mining-Techniken, einschließlich ML, die Arbeit hilfreich ergänzen. Ein zweiter Grund ist, dass viele dieser wissenschaftlichen Aufsätze auf Pre-Print-Servern liegen und nicht von Fachkollegen überprüft wurden. Die Fallstricke einer raschen Verbreitung von wissenschaftlichen Arbeiten, die nicht begutachtet wurden, wurden im Fall von Covid-19 von Yan (2020) aufgezeigt, wozu auch die Verbreitung von Verschwörungstheorien gehört. Eine Initiative zur Behebung dieses Problems wurde durch die bereits erwähnte Initiative von Publons ergriffen. Neben der Auflistung von Arbeiten und Vorabdrucken von Forschungsarbeiten im Zusammenhang mit Covid-19 hat Publons auch ein System eingeführt, mit dem Fachkollegen die aufgelisteten Arbeiten leicht bewerten und begutachten können. Die Idee ist, dass eklatant unwissenschaftliche Arbeiten auf diese Weise schnell gekennzeichnet werden.

Die Wahl der optimalen Eindämmungs- und Ausstiegspolitik wird durch einen Mangel an Daten erschwert. $\mathrm{Zu}$ den wichtigsten Unsicherheiten gehört, dass man nicht weiß, wie viele Menschen infiziert sind oder waren (Li et al. 2020); wie ansteckend und tödlich das Virus ist, auch nach dem R-Nullwert $\left(\mathrm{R}_{0}\right)$, der sich auf die durchschnittliche Zahl der Menschen bezieht, die eine infizierte Person infiziert, wenn alle dafür empfänglich sind; wie hoch die CFR ist, die das Verhältnis der Todesfälle, die Inkubationszeit und den Anteil der gefährdeten Bevölkerung angibt ( $\rho$ ) (siehe zum Beispiel Jelnov 2020, Adam 2020 und Lourenço 2020); warum manche Menschen asymptomatisch sind; und warum Kinder weniger gefährdet sind als ältere Menschen.

Trotzdem wurden auf der Grundlage vorläufiger wissenschaftlicher Erkenntnisse und epidemiologischer Modelle wichtige politische Maßnahmen getroffen, die erhebliche wirtschaftliche Auswirkungen haben. So wurde beispielsweise, wie Adam (2020) erörtert, die Entscheidung Großbritanniens, Mitte März 2020 einen strikten Lockdown und soziale Distanzierung zu verhängen, stark durch einen (nicht peer-reviewten) Aufsatz beeinflusst, der am 16. März 2020 von Forschern des Imperial College Lon- 
don veröffentlicht wurde. Dieses Papier enthielt eine schockierende Prognose: „In an unmitigated epidemic, we would predict approximately 510,000 deaths in GB and 2.2 million in the US, not accounting for the potential negative effects of health systems being overwhelmed on mortality“ (Ferguson et al. 2020, S. 7).

Die Autoren dieses Aufsatzes forderten NPI, um diese Auswirkungen zu reduzieren, wobei verschiedene Annahmen zu $R_{0}(2,0-2,6)^{8}$, CFR (0,9 Prozent) und Inkubationszeit (5,1 Tage) getroffen wurden. Auf dieser Basis empfahlen sie eine Kombination aus Fallisolierung, sozialer Distanzierung der gesamten Bevölkerung und Haushaltsquarantäne oder Schließung von Schulen und Universitäten. Obwohl es viele Unsicherheiten hinsichtlich der Wirksamkeit gebe, sei eine solche kombinierte Strategie am ehesten geeignet, um sicherzustellen, dass der Bedarf an Intensivpflegebetten innerhalb der Überlaufkapazität bleibe (Ferguson et al. 2020, S. 10).

Darüber hinaus unterstrichen die Autoren, dass es, solange es keinen Impfstoff gibt, zu einem Wiederaufflammen der Infektionsverbreitung kommen könnte, sobald die NPIs gelockert oder aufgegeben werden. Somit würde jegliche Ausstiegsstrategie davon abhängen, dass man mehr Daten erhält und mehr Forschung betreibt, um genauere Annahmen treffen zu können. Falls sich beispielsweise herausstellt, dass Covid-19 sehr ansteckend ist, aber nur eine geringe Sterblichkeitsrate aufweist, würde der Ausstiegsansatz darin bestehen, weiterhin nur diejenigen $\mathrm{zu}$ isolieren, die ein sehr hohes Risiko haben, beispielsweise alte Menschen.

Neuere Forschungen haben dazu in der Tat faszinierende Vorschläge geliefert. Razavian (2020) legt Schätzungen vor, wonach Covid-19 mit einem CFR von 0,04 Prozent 300-mal so ansteckend ist wie die saisonale Grippe, aber weitaus weniger tödlich ${ }^{9}$, und dass sich zwar etwa 96 Prozent der Bevölkerung infizieren, aber keine Symptome haben werden. Li et al. (2020) schätzen den Anteil der asymptomatischen Bevölkerung ebenfalls hoch, auf 86 Prozent. Dowd et al. (2020) stellen fest, dass das Sterblichkeitsrisiko von Covid-19 vor allem bei den über 80 -jährigen konzentriert ist. In Italien seien 96,9 Prozent der Todesfälle bei Personen über 60 Jahren aufgetreten. Unter Verwendung eines epidemiologischen Modells des Vereinigten

8 Daten aus China zeigten, dass $\mathrm{R}_{0}$ zum Zeitpunkt des Ausbruchs der Krankheit bei etwa 2,5 lag (Anderson et al. 2020). Sanche et al. (2020) schätzen $\mathrm{R}_{0}$ in neueren Berechnungen auf 5,7.

9 Wie The Economist (11. April 2020) bemerkt: „If millions of people were infected weeks ago without dying, the virus must be less deadly than official data suggest.“
Königreichs - unter der Annahme eines wesentlich geringeren Anteils der gefährdeten Bevölkerung ( $\rho=0,1$ Prozent anstelle von $\rho=1$ Prozent) und einer Infektionsausbreitung von $R_{0}=2,25$ - gehen Lourenço et al. (2020) davon aus, dass es durchaus möglich ist, dass am 19. März 2020 bereits 68 Prozent der britischen Bevölkerung betroffen gewesen sein könnten. Weil die tatsächliche $\rho$ nicht bekannt ist, betonen die Autoren jedoch die „unmittelbare“ Notwendigkeit serologischer Untersuchungen zur Bestimmung des wahren Ausmaßes der Infektion.

Zusammenfassend lässt sich sagen, dass die Wahl der NPI, deren Dauer und die zu verfolgende Ausstiegsstrategie von den Daten abhängen, um genauere Schätzungen insbesondere von $R_{0}$, CFR und $\rho$ zu erhalten. Darüber hinaus erfordert die Suche nach einer langfristigen Lösung, dass die Suche nach einem Impfstoff inmitten einer Explosion von Daten und wissenschaftlichen Details zügig vorangetrieben wird. Beide Herausforderungen, der Mangel an Daten für die Modellkalibrierung und die Notwendigkeit, sich durch riesige Datenmengen für einen Impfstoff zu wälzen, können im Prinzip von der Anwendung der KI profitieren. Im nächsten Abschnitt untersuche ich das Potenzial, die Grenzen und die Fallstricke der KI in dieser Hinsicht.

\section{KI vs. Covid-19: Pharmazeutische Interventionen ${ }^{10}$}

Die KI kann bei der Bekämpfung von Covid-19 sowohl im Hinblick auf die Unterstützung pharmazeutischer als auch nicht-pharmazeutischer Interventionen helfen. In diesem Abschnitt werden pharmazeutische Interventionen erörtert, zum Beispiel Diagnosen und Prognosen sowie die Suche nach Impfstoffen und Therapien.

\subsection{Diagnose und Prognose}

In einem frühen Survey über KI-Anwendungen im Kampf gegen Covid-19 schreiben Bullock et al. (2020), dass die KI bei der Erstellung von Diagnosen auf der Grundlage von Thorax-Röntgenaufnahmen wie Röntgenbildern und Computertomographien (CT) hilfreich sein könnte. Dadurch könnten Radiologen Zeit sparen und eine Diagnose schneller und billiger stellen als mit Standard-Covid-19-Tests

10 Dieser Abschnitt stützt sich auf Naudé 2020a, b. 
(Naudé 2020b). Zu den Initiativen, die darlegen, wie dies in der gegenwärtigen Krise erreicht werden kann, gehören Chen et al. (2020) sowie Wang und Wong (2020). Radiologen haben jedoch ihre Besorgnis darüber zum Ausdruck gebracht, dass (noch) nicht genügend Daten für die Ausbildung von KI-Modellen zur Verfügung stehen, dass der Großteil der Covid-19-Lungenbilder aus chinesischen Krankenhäusern stammt, dass diese möglicherweise einer Selektionsverzerrung unterliegen und dass die Verwendung von CT-Scans und Röntgenstrahlen die Geräte kontaminieren kann (Naudé 2020b). Darüber hinaus wurde die Genauigkeit der Diagnose von Covid-19 anhand von Röntgenbildern in Frage gestellt (Engler 2020). Das American College of Radiology hat davon abgeraten, KI als „First-Line-Test“ zu verwenden (Engler 2020).

Für die Prognose, wie eine Person vom Coronavirus betroffen sein wird, geben Yan et al. (2020) und Jiang et al. (2020) typische Beispiele. Erstere verwendeten ML zur Entwicklung eines Algorithmus zur Vorhersage des Sterblichkeitsrisikos einer infizierten Person, wobei Daten von (nur) 29 Patienten des Tongji-Krankenhauses in Wuhan verwendet wurden. Jiang et al. (2020) entwickelten eine KI, die mit 80-prozentiger Genauigkeit vorhersagen kann, welche von Covid-19 betroffene Person möglicherweise ein akutes Atemnotsyndrom (ARDS) entwickeln wird. Die Stichprobe, mit der sie ihr KI-System trainierten, ist jedoch klein (nur 53 Patienten) und auf zwei chinesische Krankenhäuser beschränkt.

Zusammenfassend lässt sich sagen, dass die Anwendung der KI zur Diagnose von Covid-19 und zur Erstellung einer Prognose für die Patienten große Forschungsanstrengungen ausgelöst hat, aber noch nicht weit verbreitet ist.

\subsection{Suche nach einem Impfstoff und Therapien}

Eine Reihe von Forschungslabors und Datenzentren haben bereits angedeutet, dass sie auf die KI zurückgreifen, um nach Therapien und nach einem Impfstoff gegen Covid-19 zu suchen. Es besteht die Hoffnung, dass die KI sowohl den Prozess der Entdeckung neuer Medikamente als auch die Umnutzung bestehender Medikamente beschleunigen kann.

So hat beispielsweise DeepMind von Google, eine Firma, die für ihren AlphaGo-Spielalgorithmus bekannt ist, die KI zur Vorhersage der Struktur der Proteine der Virusinformationen verwendet, was bei der Entwicklung neuer Medikamente nützlich sein könnte. Allerdings gesteht DeepMind ein, dass diese Strukturvorhersagen nicht expe- rimentell verifiziert wurden und dass man sich der Genauigkeit dieser Strukturen deshalb nicht sicher sein könne. ${ }^{11}$

Beck et al. (2020) berichten, dass ML festgestellt hat, dass ein bereits existierendes Medikament, Atazanavir, möglicherweise zur Behandlung von Covid-19 wiederverwendet werden könnte. Darüber hinaus identifizierten Stebbing et al. (2020) in Zusammenarbeit mit Benevolent AI, einem britischen KI-Startup-Unternehmen, das Medikament Baricitinib, das gegen rheumatoide Arthritis und Myelofibrose eingesetzt wird, als eine potenzielle Therapie für Covid-19. Dies sind nur einige Beispiele für die Verwendung der KI zur Neuverwendung vorhandener Medikamente. Eine Diskussion über weitere Beispiele bieten zum Beispiel Amanat und Krammer 2020.

Obwohl die KI sehr wahrscheinlich bei der Suche nach einem Impfstoff und nach Therapien helfen wird, bleibt es doch so, dass es trotz der Fähigkeiten der KI zur Mustererkennung mindestens ein bis zwei Jahre dauern wird, bis ein Impfstoff gefunden werden kann - wenn überhaupt ${ }^{12}$. Und dann wird es beträchtliche Ressourcen und Zeit erfordern, große Teile der Weltbevölkerung zu impfen, um eine ausreichende Immunität herbeizuführen. Es werden Entscheidungen über den Zugang zu einem solchen Impfstoff und dessen Auslieferung getroffen werden müssen.

Laut Regalado (2020) kann es mindestens 18 Monate dauern, bis ein Impfstoff zur Verfügung steht. Vanderslott et al. (2020) beschreiben den langwierigen Prozess, den potenzielle Anti-Covid-19-Medikamente durchlaufen müssen. Amanat und Krammer (2020, S. 589) kommen zu dem Schluss, dass „für SARS-CoV-2 Impfstoffe zu spät kommen könnten, um die erste Welle dieser Pandemie zu beeinflussen“.

\section{KI vs. Covid-19: Nicht-pharma- zeutische Interventionen ${ }^{13}$}

\subsection{Vorhersage und Nachverfolgung}

Es gibt einen viel zitierten Bericht, nach dem ein in Kanada ansässiges KI-Modell namens BlueDot den Ausbruch der Infektionswelle am 31. Dezember 2019 vorhersagte, bevor dies die WHO am 9. Januar 2020 tat (Kreuzhuber 2020).

11 Siehe https://tinyurl.com/wtsdagu.

12 Zum Zeitpunkt der Abfassung dieses Artikels waren nur fünf Projekte zur Erforschung eines Impfstoffs für eine Erprobung am Menschen zugelassen, siehe https://www.cbsnews.com/news/coronavirus-vaccine-covid-19-human-clinical-trial-oxford-england/.

13 Dieser Abschnitt stützt sich auch auf Naudé 2020a, b. 
Die Publicity, die BlueDot erhalten hat, ist jedoch übertrieben. Sie unterbewertet die Rolle der Wissenschaftler, die mit KI arbeiten. Zum einen haben chinesische Wissenschaftler, wie Engler (2020) hervorhebt, das Virus bereits am 26. Dezember identifiziert. Zum anderen gab im Bostoner Kinderkrankenhaus ein anderes auf KI basierendes Modell, HealthMap, am 30. Dezember 2019 eine Warnung heraus, also noch vor BlueDot. Die amerikanische HealthMap maß dem Ausbruch jedoch nur eine sehr geringe Bedeutung bei - was ein Schlaglicht darauf wirft, dass die richtige Anwendung von KI des Menschen und seines fachlichen Inputs bedarf, über Disziplingrenzen hinweg (siehe auch Hollister 2020).

Nach dem Ausbruch einer Epidemie/Pandemie kann man KI zur Vorhersage der Ausbreitung nutzen. Beispielsweise berechneten Bogoch et al. (2020) unter Verwendung von BlueDot einen Infectious Disease Vulnerability Index (IDVI), aus dem sie auf der Grundlage von Reisedaten aus Wuhan die 20 am stärksten gefährdeten Länder auflisteten. Dies war ziemlich genau bei der Vorhersage, dass Länder wie Thailand, Hongkong, Südkorea und Taiwan zuerst betroffen sein könnten. Dabei wurde jedoch völlig übersehen, wie gefährdet Länder wie Iran und Italien sind.

Ein zweites Beispiel für den möglichen Einsatz der KI zur Vorhersage der Ausbreitung eines Virus stammt aus einer früheren Pandemie, dem Zika-Virus 2015. Akhtar et al. (2019) entwickelten ein dynamisches neuronales Netzwerk, um die Ausbreitung des Virus vorherzusagen, allerdings ex post. Modelle wie diese müssen jedoch unter Verwendung von Daten aus der Covid-19-Pandemie neu trainiert werden, da sich das Corona-Virus zu sehr vom Zika-Virus und den saisonalen Grippeviren unterscheidet (Hao 2020). Zusätzlich zu diesem Mangel an historischen und unverzerrten Daten, auf denen die KI geschult werden kann, gibt es weitere Probleme, die ihren Einsatz für Vorhersage und Tracking in der gegenwärtigen Krise einschränken (Rowan 2020 und Lazer et al. 2014).

Lazer et al. (2014) verweisen auf das berüchtigte Versagen von Google Flu Trends, selbst den Ausbruch und die Ausbreitung der gewöhnlichen Grippe vorherzusagen, und bezeichnen diese als „Big-Data-Überheblichkeit und Algorithmendynamik“. In dem Maße, wie sich die Infektion weiter ausbreitet und der soziale Medienverkehr um sie herum zunimmt, nimmt auch die Menge des Lärms zu, die gefiltert werden muss, bevor sich sinnvolle Trends erkennen lassen. Allgemeiner betrachtet handelt es sich bei dem Schock und den globalen Auswirkungen von Covid-19 jedoch um ein massives Ausreißerereignis, das zu riesigen Mengen noch nie dagewesener Daten führt. Dies wird sich nachteilig auf alle Vorhersagemodelle auswirken, die historische Zeitreihendaten verwenden, einschließlich der in der Wirtschafts- und Finanzwelt verwendeten Daten. Rowan (2020) beschreibt diese Masse an Ausreißerdaten als „das Kryptonit“ der modernen KI. Daraus folgert er, dass in naher Zukunft viele Wirtschaftszweige wieder mehr auf Menschen als auf Modelle setzen werden, wenn es um Vorhersagen geht.

Aufgrund dieser Datenmängel sind nicht KI-Modelle der Stand der Technik in Bezug auf die Vorhersage und das Tracking von Covid-19, sondern etablierte epidemiologische Modelle, so genannte SIR-Modelle, abgeleitet von Kermack und McKendrick 1927. Zum Beispiel liefert das Institute for the Future of Humanity an der Universität Oxford Vorhersagen über die Ausbreitung des Virus auf der Grundlage des epidemiologischen Modells GLEAMviz ${ }^{14}$.

Das Robert-Koch-Institut in Berlin verwendet ein epidemiologisches SIR-Modell, das Eindämmungsmaßnahmen von Regierungen wie Lockdowns, Quarantänen und Vorschriften zur sozialen Distanzierung berücksichtigt. Dieses Modell wurde bereits früher im Falle Chinas verwendet, um zu veranschaulichen, dass Eindämmung erfolgreich sein kann, um die Ausbreitung auf geringere als exponentielle Raten zu reduzieren (siehe Maier und Brockmann 2020).

Ein ähnlich erweitertes SIR-Modell, das Maßnahmen des öffentlichen Gesundheitswesens gegen die Pandemie berücksichtigt und Daten aus China verwendet, wurde kürzlich von Song et al. (2020) vorveröffentlicht und anderen Forschern im $R$-Format zur Verfügung gestellt. Ferguson et al. (2020) haben in ihrer erwähnten Studie ebenfalls ein SIR-Modell des Imperial College verwendet, um ihre einflussreichen Vorhersagen über die möglichen Auswirkungen von Covid-19 in Großbritannien und den Vereinigten Staaten zu treffen.

Atkeson (2020) verwendet ein SIR-Modell zur Veranschaulichung dessen, was unter Berücksichtigung der Auswirkungen auf die öffentliche Gesundheit im Vergleich zu den wirtschaftlichen Auswirkungen von Covid-19 im Fall der Vereinigten Staaten auf dem Spiel steht. Er kommt zu dem Schluss, dass soziale Distanzierungsmaßnahmen bis zur Entwicklung eines Impfstoffs beibehalten werden müssten, räumt aber ein, dass einfache SIR-Modelle keine Antwort auf die Frage geben können, welche wirtschaftlichen Auswirkungen am stärksten sein werden: die Folgen des Lockdown oder die gesundheitliche Belastung durch die Krankheit. In diesem Zusammenhang ist eine der bisher ausgefeiltesten Modellierungsbemühungen von Eichenbaum et al. (2020) zu nennen, die ein kombiniertes makroökonomisches SIR-Modell für die Vereinigten Staa-

14 Siehe http://www.gleamviz.org. 
ten verwenden, um die Kosten verschiedener Szenarien von Lockdown und sozialer Distanzierung hinsichtlich der Auswirkungen auf die öffentliche Gesundheit zu vergleichen. Sie kommen zu dem Ergebnis, dass eine optimale Lockdown-Politik den Konsum in einem Jahr um 22 Prozent senkt und 500.000 Leben rettet.

Zusammenfassend lässt sich sagen, dass die KI noch nicht in der Lage ist, Covid-19 zuverlässig vorherzusagen und nachzuverfolgen. Stand der Technik sind etablierte epidemiologische Modelle - mit ihren Unzulänglichkeiten in Ermangelung genauer Daten, die sie benötigen, um ihre kritischsten Parameter wie Ansteckungsraten, Sterblichkeitsraten und den Prozentsatz der gefährdeten Bevölkerung zu kalibrieren. Diese Modelle können aber immerhin für eine ökonomische Kosten-Nutzen-Analyse verwendet werden, um die wirtschaftlichen Folgen der Pandemie zu minimieren (siehe zum Beispiel Eichenbaum et al. 2020).

\subsection{Soziale Kontrolle und Kontaktverfolgung ${ }^{15}$}

Zwar ist die KI noch nicht nützlich für die Vorhersage und Diagnose, und ihre Rolle bei der Suche nach einem neuen Impfstoff wird wahrscheinlich auch noch auf sich warten lassen, doch wird ihr Einsatz zur sozialen Kontrolle dramatisch zunehmen. Obgleich Datenprobleme die Vorhersage und Diagnose erschweren, ist dies keine Einschränkung für die Überwachungstechnologie, die mobile Geräte in Verbindung mit GPS- und Bluetooth-Technologie verwendet. Tatsächlich wird diese Technologie bereits seit 2011 zur Verfolgung von Ausbrüchen von Infektionskrankheiten eingesetzt (Huang et al. 2020). Neuere mobile Technologie zur Verfolgung und Überwachung integriert häufig KI/ML-Modelle, zum Beispiel zur Gesichtserkennung, zur Messung der Nähe zwischen Personen mittels Bluetooth, zur Untermauerung von Chat-Bot-Schnittstellen oder zur Verbesserung der Datengenauigkeit im Fall der Selbstanzeige von Symptomen (Sanderson 2020).

Soziale Kontrolle, beispielsweise durch Überwachung, kann in der Anfangsphase des Ausbruchs erforderlich sein, um NPI durchzusetzen, zum Beispiel Lockdown und soziale Distanzierung, und auch um infizierte Personen zu identifizieren und $\mathrm{zu}$ isolieren und den Gesundheitsbehörden bei der Planung zu helfen (Petropoulos 2020). In dieser Hinsicht ist der Einsatz von Massenüberwachung zur Durchsetzung von NPI in China gut dokumentiert (Chun 2020). Die chinesischen Behörden haben zum Beispiel in

15 Dieser Unterabschnitt stützt sich auf Naudé 2020c. öffentlichen Räumen wie Bahnhöfen installierte Infrarotkameras eingesetzt, um möglicherweise infizierte Personen zu identifizieren und die soziale Distanzierung durchzusetzen. Hongkong hat die Geofencing-Technologie zur Durchsetzung von Quarantänen eingesetzt. Aus Übersee ankommende Personen erhielten am Flughafen ein Armband mit QR-Code, das über eine App namens StayHomeSafe mit ihrem Smartphone gekoppelt wurde. Dieses verfolgte dann ihre Bewegungen und alarmierte die Behörden, wenn sie ihre Häuser verließen (Huang et al. 2020).

Die Ermittlung von Kontaktpersonen ist eine traditionelle Methode zur Bekämpfung von Epidemien und Pandemien, bei der die Gesundheitsbehörden, sobald bei einer Person ein Virus diagnostiziert wurde, alle Personen zurückverfolgen, mit denen diese Person in der zurückliegenden Zeit in Kontakt gekommen ist. Diese Personen werden dann kontaktiert und isoliert. Ferretti et al. (2020, S. 1) stellen fest, dass sich das Virus im Fall von Covid-19 zu schnell ausbreitet, als dass es durch eine herkömmliche, manuelle Kontaktverfolgung wirksam eingedämmt werden könnte. Sie modellieren den Einsatz einer App zur Kontaktverfolgung und stellen fest, dass die Pandemie eingedämmt werden könnte, wenn genügend Personen diese App nutzten, ohne dass Massenquarantänen (Lockdowns) erforderlich wären, die der Gesellschaft schaden.

Lockdowns und soziale Distanzierungsmaßnahmen können wie gesagt zwar wirksam sein, um $R_{0}$ unter $1 \mathrm{zu}$ senken, doch sie verursachen exorbitante wirtschaftliche Kosten (Atkeson 2020, Eichenbaum et al. 2020, McKibbin und Roshen 2020 sowie McNeil 2020). Da sie wirtschaftlich nicht nachhaltig sind, werden sie irgendwann auslaufen müssen - im Rahmen einer Ausstiegsstrategie. Um die Gefahr eines Wiederaufflammens der Infektionen während des Ausstiegs aus diesen Maßnahmen zu begrenzen, kann es notwendig sein, durch groß angelegte diagnostische Tests diejenigen zu identifizieren, die noch infiziert sind, und sie in Quarantäne zu halten (Dewatripont et al. 2020). Das makroökonomische SIR-Modell von Eichenbaum et al. (2020, S. 6) betont die potentielle Bedeutung einer intelligenten Eindämmungspolitik, die auf tatsächlichen Daten darüber beruht, wie viele Menschen anfällig, infiziert und genesen sind, und die Autoren kommen zu dem Schluss, dass der soziale Nutzen, diese Informationen zu sammeln und danach zu handeln, enorm sei.

Zur intelligenten Eindämmung können daher TracingApps, ergänzt durch groß angelegte diagnostische Tests, wertvoll sein. Sie können möglicherweise dazu beitragen, künftige Neuausbrüche $\mathrm{zu}$ verhindern und/oder $\mathrm{zu}$ begrenzen, wenn die Sperren gelockert werden. Eine zunehmende Zahl von Ländern, dem Beispiel von Ländern wie China, Hongkong, Singapur und Südkorea folgend, hat 
daher den Einsatz von Tracing-Apps eingeführt und untersucht. Gershgorn (2020) hat eine Liste von mindestens 25 Ländern zusammengestellt, die bis Mitte April 2020 auf Tracing- und andere Überwachungstechnologien zurückgegriffen haben.

Viele davon - nicht alle - verstoßen gegen Datenschutznormen, und eine wachsende Zahl von Ländern und Organisationen ringt mit den Trade-offs zwischen dem Bedarf an Daten und dem Schutz von Privatsphäre und Menschenrechten. In diesem Zusammenhang wurde gefordert, dass die Tracing-Apps auf einem dezentralisierten, datenminimierten und transparenten System mit angemessenen rechtlichen, technischen und administrativen Garantien basieren sollten, das zudem freiwillig ist (Simpson und Conner 2020). Es wird argumentiert, dass im Fall einer freiwilligen Nutzung das Vertrauen der Öffentlichkeit zu dem System, das in der Lage ist, den Datenschutz zu gewährleisten, unerlässlich ist, um eine ausreichend große Zahl von Menschen überhaupt für die Nutzung der App zu gewinnen - eine Voraussetzung für deren Erfolg (Ferretti et al. 2020 sowie Ienca und Vayena 2020).

Das Future of Privacy Forum hat eine Reihe bestehender Tracing-Apps und Software Development Kits (SDK) untersucht, und zwar K Health (Vereinigte Staaten), HaMagen (Israel), TraceTogether (Singapur), Covid Symptom Tracker (Großbritannien), Home Quarantine (Polen) und Pan-European Privacy-Preserving Proximity Tracing (PEPP-PT) (EU). Die Wissenschaftler kamen zu dem Schluss, dass es schwierig ist, Standortdaten vollständig zu anonymisieren. Sogar Apps, die aggregierte Standortdaten sammelten, um allgemeine Trends und Risiken auf der Makroebene für Forschungszwecke aufzudecken, könnten unbeabsichtigt heikle persönliche Informationen preisgeben (Sanderson 2020, S. 1).

Sanderson (2020) weist auch darauf hin, dass Apps, die Bluetooth nutzen, um die Nähe abzuschätzen, weniger „Privatsphären-invasiv“ sind als Apps, die GPS verwenden. In diesem Zusammenhang ist es bemerkenswert, dass Apple und Google mitgeteilt haben, sie wollten gegen Mai 2020 Smartphone-Apps auf den Markt bringen, um bei der Kontaktverfolgung zu helfen (O'Neill 2020a). Angesichts der Bedenken hinsichtlich des Datenschutzes werden diese Apps Bluetooth nutzen, um festzustellen, ob sich jemand in der Nähe einer infizierten Person aufgehalten hat. Es gibt jedoch Unzulänglichkeiten von Apps zur Kontaktverfolgung, die auf der Bluetooth-Technologie basieren (O’Neill 2020b). Eine davon ist, dass die Nähe zu einer anderen Person auf Grundlage der Signalstärke geschätzt wird. Freilich kann die Bluetooth-Signalstärke durch eine Vielzahl von Faktoren beeinflusst werden, darunter die Position des Mobiltelefons (ob es aufrecht oder seitlich gehalten wird, macht bereits einen Unterschied), Objekte und Oberflächen sowie das verwendete Betriebssystem. Das bedeutet, dass eine Tracing-App, die auf dieser Technologie basiert, zu viele Fehlalarme verursachen kann. Wie O’Neill (2020b) erörtert, besteht die einzige Möglichkeit, die Probleme der Signalstärke zu verringern, darin, ausreichende Datenmengen zu sammeln, aus denen ein KI-Modell lernen kann, die reale Entfernung unter Berücksichtigung des Kontexts des Benutzers besser abzuschätzen. Somit ist auch hier wieder ein Haupthindernis für den Einsatz der Technologie im Kampf gegen Covid-19 der Mangel an Daten.

\section{Abschließende Bemerkungen}

Innerhalb von fünf Monaten nach ihrem Ausbruch hat die Covid-19-Pandemie mindestens 210.000 Todesopfer in 185 Ländern gefordert. Sie hat dazu geführt, dass die Regierungen ihre Volkswirtschaften im Wesentlichen stillgelegt haben. Der Rückgang der wirtschaftlichen Aktivität in aller Welt innerhalb von 5 Monaten war in der jüngsten Geschichte beispiellos. Die Arbeitslosenzahlen sind dramatisch gestiegen. Allein in den Vereinigten Staaten hat die Zahl der Anträge auf Arbeitslosenunterstützung in einem Zeitraum von 5 Wochen zwischen März und April 2020 um 26 Millionen zugenommen. Es wird erwartet, dass die Weltwirtschaft im Jahr 2020 um 3 Prozent schrumpfen wird - ein Verlust von 9 Billionen Dollar. Die Welt steht nicht nur vor einer Gesundheitskrise, sondern auch vor einer Wirtschaftskrise.

Im vorliegenden Aufsatz habe ich argumentiert, dass die Politik zur Bewältigung dieser beiden Krisen auf Technologie zurückgreifen muss. Die Lösung des Problems wird darin bestehen, einen Impfstoff und Therapien $\mathrm{zu}$ finden sowie diese auf faire und gerechte Weise in der ganzen Welt zu verbreiten - was alles Zeit brauchen wird. Bis dahin muss die Ausbreitung der Krankheit verlangsamt werden. Angesichts der exorbitanten wirtschaftlichen Kosten der bisher von den meisten Ländern angewandten, relativ groben Eindämmungsmaßnahmen plädieren etliche Autoren ökonomischer Studien für intelligente Eindämmungsstrategien. Solche Strategien werden für jede Ausstiegsstrategie von entscheidender Bedeutung sein.

Die Suche nach einem Impfstoff und die praktische Umsetzung intelligenter Eindämmungsstrategien als Teil des bestehenden Lockdown können von der Künstlichen Intelligenz profitieren. Die Einschränkung von deren Nützlichkeit liegt jedoch in den Daten. Der Mangel an Daten reduziert die Möglichkeit, die KI zur Vorhersage, zum Tracking und zur Diagnose von Covid-19-Infektionen einzusetzen, aber auch zur Verbesserung der Genauigkeit von weni- 
ger stark in die Privatsphäre eingreifenden Tracing-Apps. Der Datenmangel wirkt sich auch auf die traditionellen epidemiologischen (SIR-)Modelle aus, auf denen die meisten NPI basieren. Infolgedessen besteht Ungewissheit über ihre kritischsten Parameter, wie Ansteckungsraten, Sterblichkeitsraten und der Anteil der gefährdeten Bevölkerung.

Glücklicherweise gibt es zum Zeitpunkt des Verfassens dieses Artikels eine wachsende Zahl globaler Initiativen, deren Ziel es ist, die Datenlücke zu schließen. Dazu gehören unter anderem das Covid-19 Open Research Dataset (CORD19), die öffentlichen Datensätze Covid-19 von Google und der AWS Covid-19 Data Lake von Amazon (Naudé 2020b). Alle Länder sollten sich an der Sammlung und dem Aufbau großer öffentlicher Datenbanken beteiligen, auf denen die KI trainiert werden kann. Die Kosten dafür sind gering, und der mögliche Nutzen ist angesichts des Bedarfs an unverzerrten und repräsentativen Daten über die Pandemie hoch. Es sollte auch als Investition für den Kampf gegen künftige Pandemien betrachtet werden. Neben dem Aufbau von Datenbanken für die Schulung der KI ist das groß angelegte Testen der Bevölkerung zur Verbesserung der Genauigkeit von Schlüsselparametern in epidemiologischen Modellen von entscheidender Bedeutung. Solche Informationen ergänzen den Einsatz von Tracing-Apps stark.

Dass der Bedarf an besseren Daten im Kampf gegen Covid-19 so wichtig ist, wirft die Frage nach dem Datenschutz und verbundenen bürgerlichen Freiheiten auf. Einige Kommentatoren verstehen dies als einen Trade-off zwischen den Belangen der öffentlichen Gesundheit und dem Datenschutz. Dieser Aufsatz sollte gezeigt haben, dass die Dinge komplexer sind: Es ist auch ein Trade-off zwischen Datenschutz und wirtschaftlichen Kosten. Wie hoch ist der wirtschaftliche Preis, den die Menschen bereit sind, für Datenschutz zu zahlen? Und gibt es überhaupt einen unvermeidlichen Trade-off? Ist es möglich, ein System für die Sammlung und Nutzung von Daten zum Zwecke des Ausstiegs aus dem Lockdown so zu konzipieren, dass der Datenschutz gewahrt bleibt? Wie wird ein solches System das Vertrauen der Bevölkerung gewinnen und halten? Diese grundlegenden Fragen wurden schon lange vor dem Ausbruch der Covid-19-Pandemie als wichtig erkannt und sind in den meisten Vorschlägen für eine angemessene Steuerung der KI enthalten. Die Pandemie hat die Beantwortung dieser Fragen dringlich gemacht.

Die KI kann bei der Suche nach einem Impfstoff und nach Therapien eine wichtige Rolle spielen. Unglücklicherweise haben die meisten Länder jedoch keinen $\mathrm{Zu}$ gang zu den fortgeschrittenen Möglichkeiten der KI.Mehr als 90 Prozent aller Investitionen in Forschung und Entwicklung sowie in KI-Risikokapital entfallen auf drei Re- gionen: Nordamerika, Europa und China. Wenn man sich einig ist, dass ein Impfstoff gegen SARS-CoV-2 ein globales öffentliches Gut ist, dann kann die KI, wenn sie hierzu beiträgt, als ein „single-best effort public good“ betrachtet werden (Barrett, 2007). Dies ist ein öffentliches Gut, das von einem oder einigen wenigen Ländern, die dazu in der Lage sind, zum Nutzen aller produziert werden kann. Die Voraussetzung dafür, dass es ein öffentliches Gut bleibt, besteht darin, dass es in einer nicht ausschließbaren und nicht rivalisierenden Weise verfügbar ist. Das bedeutet, dass bei der Mobilisierung der KI in der Suche nach einem Impfstoff ein unkoordiniertes „KI-Wettrüsten“ zwischen den Ländern vermieden werden muss und dass es klare und faire Regeln für die Verteilung und den Zugang zu einem solchen Impfstoff geben sollte. Herauszufinden, wie ein solches Ergebnis finanziert und mit Hilfe von Anreizen herbeigeführt werden kann, ist eine weitere dringende Herausforderung für Ökonomen, nicht zuletzt im Hinblick auf die Überwindung von Produktions- und Logistikproblemen (Amanat und Krammer 2020).

Die Künstliche Intelligenz kann insofern im Kampf gegen Covid-19 möglicherweise eine wertvolle Rolle spielen, als sie hilft, die wirtschaftlichen und gesundheitlichen Kosten zu senken. Wenn sie jedoch nicht richtig gehandhabt wird, wenn politische Fehler unterlaufen, dann kann sie der Volkswirtschaft auch hohe, langfristige Kosten in Form von höherer Ungleichheit, langsamer wirtschaftlicher Erholung und geringerer Dynamik aufbürden. Sie kann die wahrscheinlichen schädlichen strukturellen Folgen der Pandemie verstärken, beispielsweise die beschleunigte Automatisierung der Produktion, die Rückverlagerung der wirtschaftlichen Aktivitäten aus dem Ausland und die Konsolidierung der Marktmacht großer digitaler Plattformunternehmen.

Danksagung: Ich möchte Karen Horn herzlich für ihre sehr hilfreiche und professionelle Überarbeitung dieses aus dem Englischen übertragenen Manuskripts danken. Selbstverständlich bin ich für alle Fehler und Ungenauigkeiten selbst verantwortlich.

\section{Literaturverzeichnis}

Adam, D. (2020), Modelling the impact: The simulations driving the world's response to Covid-19, Nature 580, S. 316-18.

Akhtar, M., M. Kraemer und L. Gardner (2019), A dynamic neural network model for predicting risk of Zika in real-time, $B M C$ Medicine 17 (171).

Amanat, F. und F. Krammer (2020), SARS-CoV-2 vaccines: Status report, Immunity 52(4), S. 583-89. 
Anderson, R. M., H. Heesterbeek, D. Klinkenberg und T. D. Hollingsworth (2020), How will country-based mitigation measures influence the course of the Covid-19 epidemic? The Lancet, 395, S. 931-34.

Atkeson, A. (2020), What will be the economic impact of Covid-19 in the US? Rough estimates of disease scenarios, NBER Working Paper 26867.

Ballester, J., J. M. Robine, F. R. Herrmann und X. Rodó (2019), Effect of the Great Recession on regional mortality trends in Europe, Nature Communications 10(1), 679.

Barrett, S. (2007), Why Cooperate? The Incentive to Supply Global Public Goods, Oxford, Oxford University Press.

Beck, B., B. Shin, Y. Choi, S. Park und K. Kang (2020), Predicting commercially available antiviral drugs that may act on the novel Coronavirus (2019-nCoV), Wuhan, China, through a drug-target interaction deep learning model, bioRxiv, 2. Februar.

Bogoch, I., A. Watts, A. Thomas-Bachli, C. Huber, M. Kraemer und K. Khan (2020), Pneumonia of unknown aetiology in Wuhan, China: Potential for international spread via commercial air travel, Journal of Travel Medicine 27(2), S. 1-3.

Bullock, J., A. Luccioni, K. H. Pham, C. S. N. Lam und M. Luengo-Oroz (2020), Mapping the landscape of artificial intelligence applications against Covid-19, ArXiv, online verfügbar unter https:// arxiv.org/abs/2003.11336v1.

Chen, J. et al. (2020), Deep learning-based model for detecting 2019 novel Coronavirus pneumonia on high-resolution computed tomography: A prospective study, medRxiv, 1. März.

Chun, A. (2020), In a time of coronavirus, Chinas investment in Al is paying off in a big way, South China Morning Post vom 18. März.

Dewatripont, M., M. Goldman, E. Muraille und J.-P. Platteau (2020), Rapidly identifying workers who are immune to Covid-19 and virus-free is a priority for restarting the economy, VoxEU.org.

Dowd, J. B. et al. (2020), Demographic science aids in understanding the spread and fatality rates of Covid-19, Proceedings of the National Academy of Sciences.

Eichenbaum, M. S., S. Rebelo und M. Trabandt (2020), The macroeconomics of epidemics, NBER Working Paper 26882.

Engler, A. (2020), A guide to healthy skepticism of artificial intelligence and coronavirus, The Brookings Institution, verfügbar unter https://www.brookings.edu/research/a-guide-to-healthyskepticism-of-artificial-intelligence-and-coronavirus/.

Farboodi, M., R. Mihet, T. Philippon und L. Veldkamp (2019), Big data and firm dynamics, NBER Working Paper 25515.

Feldstein, S. (2019), The global expansion of Al surveillance, Carnegie Endowment for International Peace Working Paper.

Ferguson et al. (2020), Report 9: Impact of non-pharmaceutical interventions (NPIs) to reduce Covid-19 mortality and healthcare demand, Spiral, online verfügbar unter https://spiral.imperial. ac.uk:8443/handle/10044/1/77482.

Ferretti, L. et al. (2020), Quantifying SARS-CoV-2 transmission suggests epidemic control with digital contact tracing, Science, 31. März.

Gershgorn, D. (2020), We mapped how the Coronavirus is driving new surveillance programs around the world, Medium: OneZero, 13. April.

Grünwald, E., D. Antons und T. Salge (2020), Covid-19 Evidence Navigator, Institute for Technology and Innovation Management, RWTH Aachen.

Gopinath, G. (2020), The great lockdown: Worst economic downturn since the Great Depression, IMFBlog, 14. April.
Hinton, G., S. Osindero und T.-W. The (2006), A fast learning algorithm for deep belief nets, Neural Computation 18, S. 1527-54.

Hinton, G. und R. Salakhutdinov (2006), Reducing the dimensionality of data with neural networks, Science 313, S. 504-07.

Hollister, M. (2020), Al can help with the Covid-19 crisis - but the right human input is key, World Economic Forum, 30. März.

Huang, Y., M. Sun und Y. Sui (2020), How digital contact tracing slowed Covid-19 in East Asia, Harvard Business Review, 15. April.

lenca, M. und E. Vayena (2020), On the responsible use of digital data to tackle the Covid-19 pandemic, Nature Medicine, 27. März.

Jelnov, P. (2020), Confronting Covid-19 myths: Morbidity and mortality, GLO Discussion Paper 516.

Jiang, X. et al. (2020), Towards an Artificial Intelligence framework for data-driven prediction of Coronavirus clinical severity, Computers, Materials and Continua 63(1), S. 537-51.

Kermack, W. O. und A. G. McKendrick (1927), A contribution to the mathematical theory of epidemics, Proceedings of the Royal Society of London A 115 (772), S. 700-21.

Kreuzhuber, K. (2020), How Al, big data and machine learning can be used against the Coronavirus, ARS Electronica Blog, 19. März.

Lazer, D., R. Kennedy, G. King und A. Vespignani (2014), The parable of Google flu: Traps in big data analysis, Science 343(6176), S. 1203-05.

Li, R. et al. (2020), Substantial undocumented infection facilitates the rapid dissemination of novel Coronavirus (SARS-CoV2), Science, 16. März.

Lourenço, J. et al. (2020), Fundamental principles of epidemic spread highlight the immediate need for large-scale serological surveys to assess the stage of the SARS-CoV-2 epidemic, medRxiv preprint.

Maier, B. und D. Brockmann (2020), Effective containment explains sub-exponential growth in confirmed cases of recent Covid-19 outbreak in mainland China, medRxiv, 20. Februar.

McKibbin, W. und F. Roshen (2020), The global macroeconomic impacts of Covid-19: Seven scenarios, Brookings Institution, online verfügbar unter https://www.brookings.edu/research/ the-global-macroeconomic-impacts-of-Covid-19-seven-scenarios/.

McNeil, D. (2020), Restrictions are slowing Coronavirus infections, new data suggest, The New York Times vom 30. März.

Moor, J. (2006), The Dartmouth College Artificial Intelligence conference: The next fifty years, Al Magazine 27(4), S. 87-91.

Naudé, W. (2019), The race against the robots and the fallacy of the giant cheesecake: Immediate and imagined impacts of Artificial Intelligence, IZA Discussion Paper 12218.

Naudé, W. (2020a), Artificial Intelligence vs. Covid-19: Limitations, constraints and pitfalls, Al \& Society, 28. April.

Naudé, W. (2020b), Artificial Intelligence against Covid-19: An early review, IZA Discussion Paper 13110.

Naudé, W. (2020c), Artificial Intelligence, Covid-19, and developing countries: Priorities and trade-offs, UNU-WIDER Background Note.

New Scientist (2017), Machines that Think, London, John Murray Learning.

O'Neill, P. H. (2020a), Apple and Google are building coronavirus tracking into iOS and Android, MIT Technology Review, 10. April.

O’Neill, P. H. (2020b), Bluetooth contact tracing needs bigger, better data, MIT Technology Review, 22. April.

Petropoulos, G. (2020), Artificial Intelligence in the fight against Covid-19, Bruegel, 23. März. 
Razavian, A. S. (2020), When would Covid19 be over from a data science perspective, and what's the fatality rate? Medium, 6. April.

Reardon, J. et al. (2019), 50 ways to leak your data: An exploration of apps' circumvention of the Android permissions system, login Usenix Mag. 44.

Regalado, A. (2020), A Coronavirus vaccine will take at least 18 months if it works at all, MIT Technology Review, 10. März.

Ross, C. und R. Robbins (2020), Debate flares over using Al to detect Covid-19 in lung scans, StatPlus, 30. März.

Rowan, I. (2020), What happens to Al when the world stops (Covid19)?, Medium: Towards Data Science, 31. März.

Sanche S. et al. (2020), High contagiousness and rapid spread of severe acute respiratory syndrome coronavirus 2, Emerging Infectious Diseases 26(7).

Sanderson, P. (2020), Privacy and pandemics: The role of mobile apps, Future of Privacy Forum, 22. April.

Silver, D. et al. (2018), A general reinforcement learning algorithm that masters chess, shogi, and Go through self-play, Science 362(6419), S. 1140-44.

Simpson, E. und A. Conner (2020), Digital contact tracing to contain the Coronavirus, Center for American Progress, 22. April.
Song, P. et al. (2020), An epidemiological forecast model and software assessing interventions on Covid-19 epidemic in China, medRxiv, 3. März.

Stebbing, J. et al. (2020), Covid-19: Combining antiviral and antiinflammatory treatments, The Lancet 20, S. 400-01.

Tegmark, M. (2017), Life 3.0: Being Human in the Age of Artificial Intelligence, New York, Knopf Doubleday Publishing Group.

Vanderslott, S., A. Pollard und T. Thomas (2020), Coronavirus vaccine: Here are the steps it will need to go through during development, The Conversation, 30. März.

Wang, L. und A. Wong (2020), Covid-Net: A tailored deep convolutional neural network design for detection of Covid-19 cases from chest radiography images, arXiv, 22. März.

World Intellectual Property Organization - WIPO (2019), Technology Trends 2019: Artificial Intelligence, Genf.

Yan, W. (2020), Coronavirus tests science's need for speed limits, New York Times vom 14. April (mit Update am 21. April).

Yan, L. et al. (2020), Prediction of criticality in patients with severe Covid-19 infection using three clinical features: A machine learning-based prognostic model with clinical data in Wuhan, medRxiv, 3. März. 